\title{
The behaviour of measles transmission in three different populations
}

\author{
Budi Priyo Prawoto*, Dimas Avian Maulana, and Yuliani Puji Astuti \\ Universitas Negeri Surabaya, Department of Mathematics, Surabaya, Indonesia
}

\begin{abstract}
SIR Model can be employed to model the transmission of either fatal or non-fatal disease within a closed population based on certain assumptions. In this paper, the behaviour of non-fatal diseases transmission model is observed from three types of population, that is (i) increasing population, (ii) constant population, (iii) decreasing population. This paper acquired two equilibria, i.e, the disease-free equilibrium point $\left(\frac{\mu}{\mu_{5}}, 0,0\right)$ and the endemic equilibrium point $\left(\frac{\alpha+\mu_{I}}{\beta}, \frac{\mu \beta-\mu_{5} \alpha-\mu_{5} \mu_{I}}{\beta\left(\alpha+\mu_{I}\right)}, \frac{\alpha\left(\mu \beta-\mu_{5} \alpha-\mu_{5} \mu_{1}\right)}{\beta\left(\alpha+\mu_{I}\right) \mu_{R}}\right)$. At the disease-free equilibrium, the behaviour of the model is stable when $\beta<\frac{\mu_{5}}{\mu}\left(\mu_{I}+\alpha\right)$, while at the endemic equilibrium, its behaviour is stable for any positive parameters $\alpha, \beta, \mu, \mu_{\mathrm{S}}, \mu_{\mathrm{I}}$ and $\mu_{\mathrm{R}}$.
\end{abstract}

\section{Introduction}

Each year through surveillance activities reported more than 11,000 cases of measles suspects and results laboratory confirmation shows $12-39 \%$ of which are measles for sure (lab confirmed) while $16-43 \%$ is definite rubella [1]. From 2010 to 2015 , it is estimated 23,164 cases of measles and 30,463 cases of rubella. The number of cases is estimated to be lower compared to actual figures in the field, given the number of unreported cases, especially from private health services and the completeness of surveillance reports that are still low.

The mathematical model becomes a powerful tool that eases the mathematization of the real-life problem using certain criteria and assumptions [2]. In the next step, the solution to the governing equation can be addressed, both analytical and numerical solutions.

One of the real-world problems is the transmission of a non-fatal disease i.e. measles, influenza, and the others. Over the years, childhood diseases are the most common form of infectious diseases. Since children are in particularly close contact with their peers, such diseases can spread quickly. The infected individuals will have immunity in a certain period. This transmission problem then converted to a mathematical SIR Model.

SIR Model which stands for Susceptible, Infective, Recovered originally developed to describe the transmission and extinction of an epidemic disease outbreak in the closed population. In this paper, we discussed the establishment of a non-fatal SIR model based on the assumptions. Once the model is formed, the analytical solutions and the equilibrium point is searched, which further interpreted, the behaviour of the disease and its existence, which is disease-free equilibrium and endemic equilibrium.
The stability of measles transmission model with and without vaccination at constant population was studied by Prawoto in 2017. In term of population with vaccination, there are $T_{1}=(1,0) \quad$ and $T_{2}=\left(\frac{\mu+\alpha}{\beta},-\frac{\mu(-\beta+\mu+\alpha)}{\beta(\mu+\alpha)}\right)$ as the stationary points, while in population without vaccination, there are $T_{3}=(1-\varepsilon, 0)$ and $T_{4}=\left(\frac{\mu+\alpha}{\beta},-\frac{\mu(-(1-\varepsilon) \beta+\mu+\alpha)}{\beta(\mu+\alpha)}\right)$ as the stationary points [3]. Prawoto had analysed that the model is stable around $T_{1}$ hold by $\beta<\mu+\alpha$, and it would be stable around $T_{2}$ when $\beta>\mu+\alpha$. In the second population, the model would be stable around $T_{3}$ and $T_{4}$ when $\beta<\frac{\mu+\alpha}{1-\varepsilon}$ and $\beta>\frac{\mu+\alpha}{1-\varepsilon}$ respectively.

\section{SIR Model}

Non-fatal disease transmission, such as influenza, measles, etc, in a population, is assumed to have a fixed amount and a period of the outbreak. For example, at a certain time $t$, in a population consisting of:

- $s(t)$, susceptible: a subpopulation of those members consists of people who are susceptible to the disease.

- $I(t)$, infective: a subpopulation of those members consists of people who have contracted the disease.

- $R(t)$, recovered: people who have been cured of the disease.

With $\bar{S}(t)+\bar{I}(t)+\bar{R}(t)=N(t)$, where $N(t)$ is the number of total population, and the proportion of $\frac{S(t)}{N(t)}+\frac{\Gamma(t)}{N(t)}+\frac{\pi(t)}{N(t)}=S+I+R=1$. [2]

The compartment we put together is as follows:

* Corresponding author: budiprawoto@unesa.ac.id 


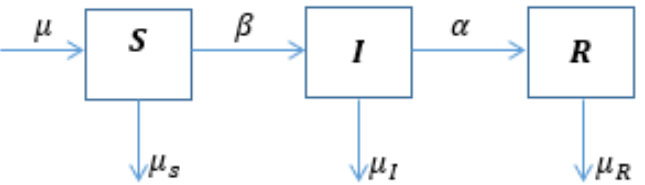

The mathematical model of the diagram above is.

$$
\begin{aligned}
& \frac{d S}{d t}=\mu-\mu_{s} s-\beta S I \\
& \frac{d I}{d t}=\beta S I-\mu_{I} I-\alpha I \\
& \frac{d R}{d t}=\alpha I-\mu_{R}
\end{aligned}
$$

$S(t)$ is the number of vulnerable populations the disease $I(t)$ is the number of the population is infected

$R(t)$ is a population recovering from illness $\mu$ is the rate of natality

$\mu_{s}$ is the rate of mortality at $S$

$\mu_{I}$ is the rate of mortality in $I$

$\mu_{R}$ is the rate of mortality in $R$

$\beta$ is the rate of disease transmission

$\alpha$ is the rate of recovery

To obtain the equilibrium of system [1], the right-hand side must equal zero.

$$
\begin{aligned}
& \mu-\mu_{s} s-\beta S I=0 \\
& \beta S I-\mu_{I} I-\alpha I=0
\end{aligned}
$$

Since the last equation has no effect on the two previous equations, then it can be ignored first. We obtained two equilibria $(S, I, R)$, which are:

$$
T_{1}=\left(\frac{\mu}{\mu_{s}}, 0,0\right)
$$

And

$$
T_{2}=\left(\frac{\alpha+\mu_{I}}{\beta}, \frac{\mu \beta-\mu_{S} \alpha-\mu_{S} \mu_{I}}{\beta\left(\alpha+\mu_{I}\right)}, \frac{\alpha\left(\mu \beta-\mu_{S} \alpha-\mu_{S} \mu_{I}\right)}{\beta\left(\alpha+\mu_{I}\right) \mu_{R}}\right)
$$

To do the stability analysis of system [1], a linearization is needed because the system [1] is not a linear system. This process allows us to zoom in the behaviour of the model around the equilibria. For instance,

$f(S, I, R)=\mu-\mu_{s} S-\beta S I$

$g(S, I, R)=\beta S I-\mu_{I} I-\alpha I$

Then

$f_{s}=-\mu_{s}-\beta I$

$f_{1}=-\beta S$

$g_{s}=\beta I$

$g_{1}=\beta S-\mu_{l}-\alpha$

The Jacobian matrix is

$I=\left(\begin{array}{cc}-\mu_{s}-\beta I & -\beta S \\ \beta I & \beta S-\mu_{I}-\alpha\end{array}\right)$

For the equilibrium $T_{1}=\left(\frac{\mu}{\mu_{s}}, 0,0\right)$, the Jacobian matrix is given:

$$
\begin{gathered}
I=\left(\begin{array}{cc}
-\mu_{s} & -\beta \frac{\mu}{\mu_{s}} \\
0 & \beta \frac{\mu}{\mu_{s}}-\mu_{I}-\alpha
\end{array}\right) \\
\left|\lambda I_{M}-J\right|=\left|\begin{array}{cc}
\lambda+\mu_{s} & \beta \frac{\mu}{\mu_{s}} \\
0 & \lambda-\left(\beta \frac{\mu}{\mu_{s}}-\mu_{I}-\alpha\right)
\end{array}\right|=0, I_{M} \text { is } \\
\text { Identity Matrix of } 2 \times 2 . \\
\left|\lambda I_{M}-J\right|=\left(\lambda+\mu_{s}\right)\left(\lambda-\left(\beta \frac{\mu}{\mu_{s}}-\mu_{I}-\alpha\right)\right)=0
\end{gathered}
$$

We obtained $\lambda_{1}=-\mu_{s}$ or $\lambda_{2}=\beta \frac{\mu}{\mu_{s}}-\mu_{1}-\alpha$.

To make the system is stable, it should be $\lambda<0$,

$\lambda_{1}$ is negative due to $\mu_{s}>0$, for $\lambda_{2}<0$ then $\beta<\frac{\mu_{5}}{\mu}\left(\mu_{1}+\alpha\right)[4]$.

Simulation case 1 (the natality is smaller than the mortality) (Fig. 1).

Taking

$\mu=0.01, \mu_{s}=\mu_{1}=\mu_{R}=0.02, \alpha=0.1$ and

$\beta=0.01\left(<\frac{\mu_{s}}{\mu}\left(\mu_{1}+\alpha\right)\right)$, we obtained the equilibrium $T_{1}=(0.5,0,0)$.

Simulation case 2 (the natality is equal to the mortality) (Fig. 2).

Taking

$\mu=0.02, \mu_{s}=\mu_{1}=\mu_{R}=0.02, \alpha=0.1$ and

$\beta=0.08\left(<\frac{\mu_{S}}{\mu}\left(\mu_{1}+\alpha\right)\right)$, we obtained the equilibrium $T_{1}=(1,0,0)$.

Simulation case 3 (the natality is higher than the mortality) (Fig. 3)

Taking

$\mu=0.06, \mu_{s}=\mu_{1}=\mu_{R}=0.02, \alpha=0.1$ and

$\beta=0.02\left(<\frac{\mu_{s}}{\mu}\left(\mu_{1}+\alpha\right)\right)$.

we obtained the equilibrium $T_{1}=(3,0,0)$.

The figure 1 to figure 3 of the simulation is given respectively as follows:

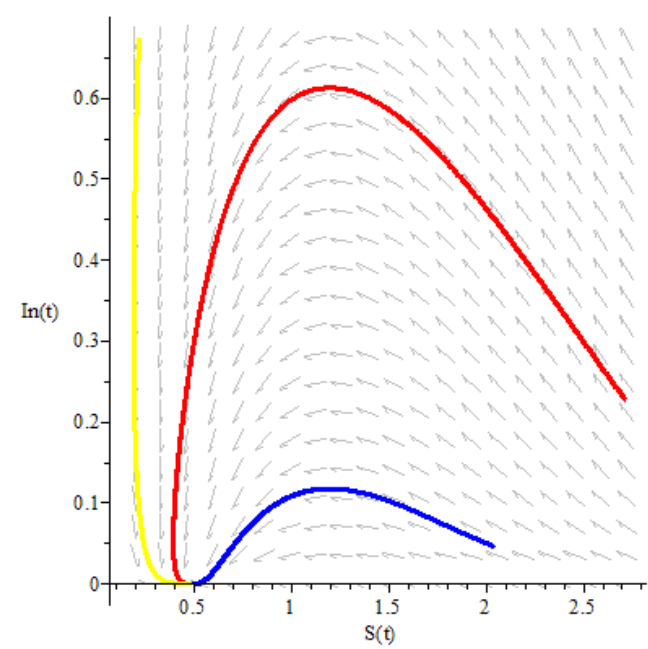

Fig. 1. The behaviour of Model around $T_{1}=(0.5,0,0)$ 


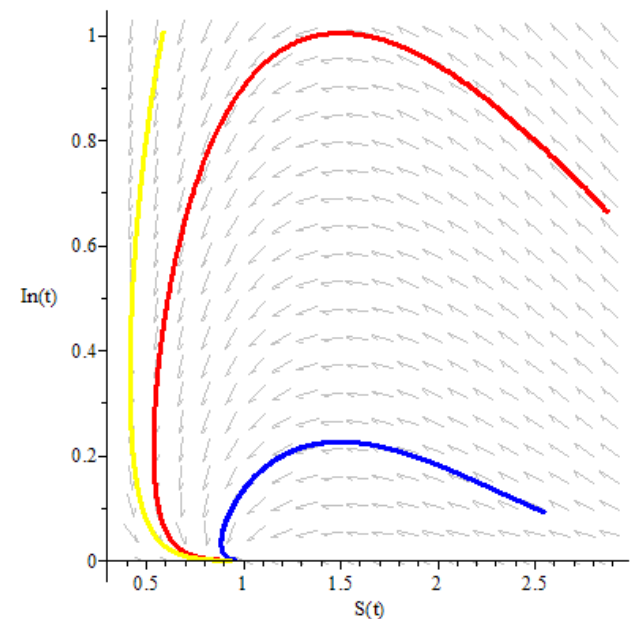

Fig. 2. The behaviour of Model around $T_{1}=(1,0,0)$

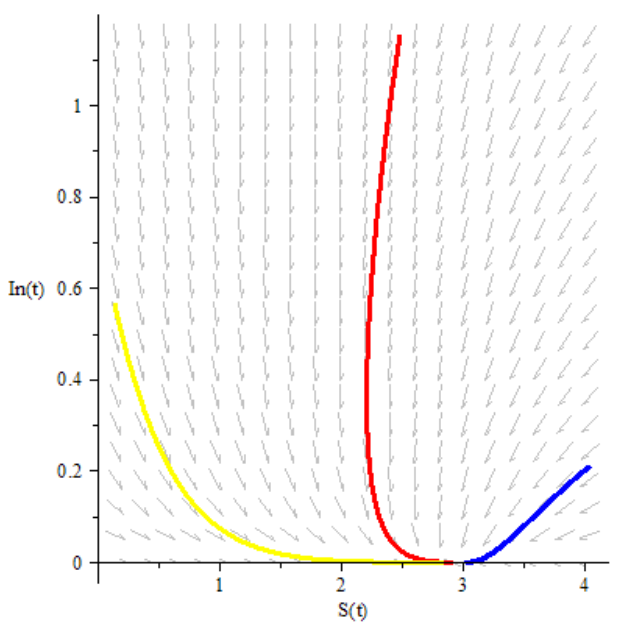

Fig. 3. The behaviour of Model around $T_{1}=(3,0,0)$

Figure 1 shows that the behaviour of the model tends to point $(0.5,0,0)$, with the given parameter values, wherever the initial population pointed. Similar to fig. 1, figure 2 and figure 3 shows that the behaviour of the model tends to point $(1,0,0)$ and $(3,0,0)$ respectively. It means that for $t \rightarrow \sim$ the value of $S, I$ and $R$ is convergent to a certain point.

For the equilibrium point $T_{2}=\left(\frac{\alpha+\mu_{I}}{\beta}, \frac{\mu \beta-\mu_{5} \alpha-\mu_{5} \mu_{I}}{\beta\left(\alpha+\mu_{i}\right)}, \frac{\alpha\left(\mu \beta-\mu_{5} \alpha-\mu_{S} \mu_{I}\right)}{\beta\left(\alpha+\mu_{I}\right) \mu_{R}}\right), \quad$ we also obtained the Jacobian matrix:

$I=\left(\begin{array}{cc}-\frac{\mu \beta}{\alpha+\mu_{l}} & -\left(\alpha+\mu_{l}\right) \\ \frac{\mu \beta}{\left(\alpha+\mu_{l}\right)}-\mu_{s} & 0\end{array}\right)$

$\left|\lambda I_{M}-J\right|=\left|\begin{array}{cc}\lambda+\frac{\mu \beta}{\alpha+\mu_{I}} & \alpha+\mu_{l} \\ \mu_{S}-\frac{\mu \beta}{\left(\alpha+\mu_{l}\right)} & \lambda\end{array}\right|=0$

$\left|\lambda I_{M}-J\right|=\lambda\left(\lambda+\frac{\mu \beta}{\alpha+\mu_{I}}\right)-\left(\left(\alpha+\mu_{I}\right) \mu_{s}-\mu \beta\right)=0$.
We acquired

$\lambda_{1,2}=\frac{1}{2}\left(-\frac{\mu \beta}{\alpha+\mu_{I}} \pm \sqrt{\left(\frac{\mu \beta}{\alpha+\mu_{I}}\right)^{2}-\left(4\left(\alpha+\mu_{l}\right) \mu_{s}+\mu \beta\right)}\right)$

To make the system is stable, it should be $\lambda<0$ or $R_{e}(\lambda)<0$.

$\lambda_{1,2}$ will be all negative when $\left(\frac{\mu \beta}{\alpha+\mu_{1}}\right)^{2} \geq\left(4\left(\alpha+\mu_{1}\right) \mu_{s}+\mu \beta\right)$. When $\left(\frac{\mu \beta}{\alpha+\mu_{I}}\right)^{2}<\left(4\left(\alpha+\mu_{1}\right) \mu_{S}+\mu \beta\right)$ then $\lambda_{1,2}$ are complex numbers and the real part is negative $\left(R e\left(\lambda_{1,2}\right)=-\frac{1}{2} \frac{\mu \beta}{\alpha+\mu_{I}}<0\right)$, then for all positive parameter $\alpha, \beta, \mu, \mu_{s}, \mu_{1}$ and $\mu_{R}$ the system is stable.

Simulation case 1 (the natality is smaller than the mortality) (Fig. 4)

Taking

$\mu=0.01, \mu_{s}=\mu_{l}=\mu_{R}=0.02, \alpha=0.1$ and

$\beta=0.8$, we obtained the equilibrium $T_{2}=(0.15,0.0583,0.2916)$.

Simulation case 2 (the natality is equal to the mortality) (Fig. 5)

Taking

$\mu=0.02, \mu_{s}=\mu_{1}=\mu_{R}=0.02, \alpha=0.1$ and

$\beta=0.3$, we obtained the equilibrium

$T_{2}=(0.4,0.1,0.5)$.

Simulation case 3 (the natality is higher than the mortality) (Fig. 6)

Taking

$\mu=0.06, \mu_{s}=\mu_{I}=\mu_{R}=0.02, \alpha=0.1$ and

$\beta=0.1$, we obtained the equilibrium $T_{2}=(1.2,0.3,1.5)$.

The figure 4 to figure 6 of the simulation is given respectively as follows:

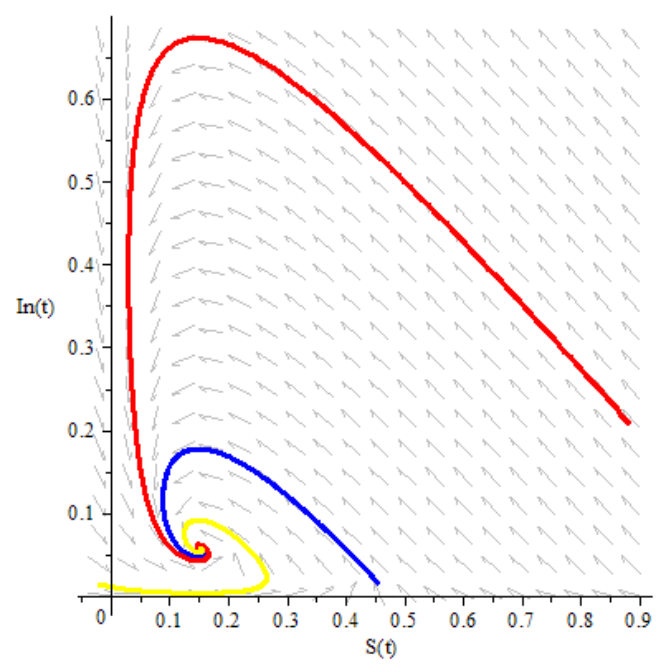

Fig. 4. The behaviour of Model around $T_{2}=(0.15,0.0583,0.2916)$ 


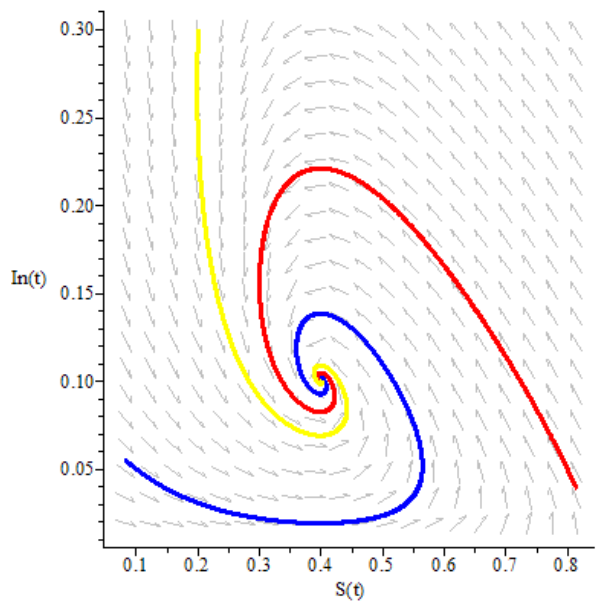

Fig. 5. The behaviour of Model around $T_{2}=(0.4,0.1,0.5)$

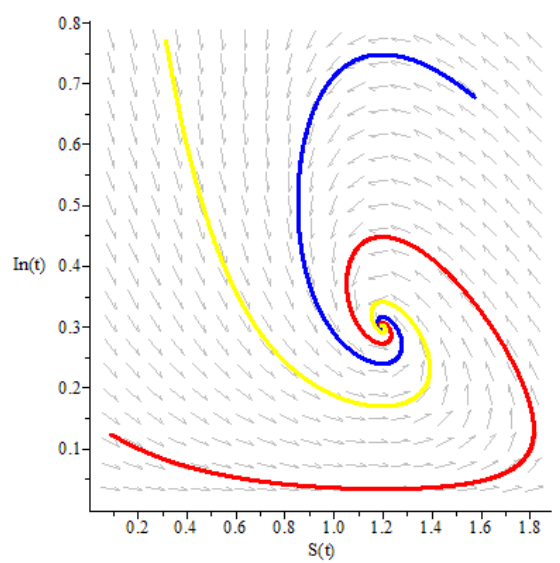

Fig. 6. The behaviour of Model around $T_{2}=(1.2,0.3,1.5)$

\section{Conclusion}

It is acquired two equilibria, that are: $T_{1}=\left(\frac{\mu}{\mu_{s}}, 0,0\right)$ and $T_{2}=\left(\frac{\alpha+\mu_{I}}{\beta}, \frac{\mu \beta-\mu_{S} \alpha-\mu_{S} \mu_{I}}{\beta\left(\alpha+\mu_{I}\right)}, \frac{\alpha\left(\mu \beta-\mu_{S} \alpha-\mu_{S} \mu_{I}\right)}{\beta\left(\alpha+\mu_{I}\right) \mu_{R}}\right)$. The first equilibrium is called a disease-free equilibrium because the infected population is 0 . The second equilibrium is called endemic equilibrium because the population is infected with the positive.

The stability of the disease-free equilibrium occurs if $\beta<\frac{\mu_{s}}{\mu}\left(\mu_{1}+\alpha\right)$. While the stability of the endemic equilibrium holds for all the positive parameter values $\alpha, \beta, \mu, \mu_{s}, \mu_{l}$ and $\mu_{R}$.

The authors thank the Universitas Negeri Surabaya for the financial support and the anonymous referees for their valuable suggestions and comments.

\section{References}

1. World Health Organisation. World Health Organisation Regional Office for South-East Asia. www.searo.who.int. [Online] [Cited: 30 April 2018.]

http://www.searo.who.int/indonesia/topics/immuniz ation/mr_measles_status.pdf?ua $=1$.
2. O. Diekmann \& Heesterbeek. Mathematical Epidemiology of Infectious Diseases: Model Building, Analysis and Interpretation. (New York: John Wiley, 2000)

3. Stability and Simulation of Measles Transmission Model with and without Vaccination. Prawoto, Budi Priyo. 2, Allahabad: Puspha Publishing House, Vol. 102. 0972-0871. (2017)

4. Analytical Approximate Solution of a SIR Epidemic Model with Constant Vaccination Strategy by Homotopy Perturbation Method. Yildirim, Ahmet. Emerald Insight, pp. 1566-1575. (2009) 